ORIGINAL ARTICLE

\title{
Olfactometric assessment - where do we stand today?
}

\author{
Violeta Melinte ${ }^{1,2,3}$, Codrut Sarafoleanu ${ }^{1,2,3}$ \\ 1"Carol Davila" University of Medicine and Pharmacy, Bucharest, Romania \\ ${ }^{2}$ CESITO Centre, "Sfanta Maria" Hospital, Bucharest, Romania \\ ${ }^{3}$ ENT\&HNS Department, "Sfanta Maria” Hospital, Bucharest, Romania
}

\section{ABSTRACT}

Having an enormous importance not only in one's quality of life, but also in one's health and personal safety, the olfactory function assessment has begun to gain more and more interest amongst the ENT practitioners. However, at the moment, there is no worldwide accepted evaluation protocol available despite the fact that studies regarding smell disorders and their evaluation have been published all over the world.

The purpose of this article is to present the olfactory assessment methods practiced today not only in different clinics from Europe and the USA, but also in Romania, because we have recently started to study the olfactory function disturbances. Three of the most interesting clinical cases, assessed in our ENT Department of the "Sfanta Maria" Clinical Hospital between 2015 and 2016 , will be discussed in the current paper.

KEYWORDS: olfactometry, smell disorders, n-butanol, olfactory function, odor

\section{INTRODUCTION}

Despite the well-known sense of smell importance, the olfactory evaluation methods are still far away from being organized in a general international protocol. Specialty literature describes many assessment means, but unfortunately most of them still have limited clinical applicability.

Worldwide, there is no consensus in what the olfactory assessment is concerned, if psychophysical evaluations are enough in order to diagnose smell disorders or if the results would be better or clearer if electrophysiological measurements were added.

This article discusses the olfactory assessment methods currently used in Western Europe, in the USA and also in Romania. Three clinical cases of anosmia assessed in "Sfanta Maria" ENT Department between 2015 and 2016 will be presented in order to illustrate our approach regarding the olfactory disorders. The particularities of diagnosis and evaluation are presented together with a short literature review regarding smell impairment.

\section{OLFACTORY FUNCTION ASSESSMENT - BRIEF LITERATURE REVIEW}

Currently, various techniques for the investigation of chemosensory functions are available.
They are divided into psychophysical and electrophysiological methods. The psychophysical measures are represented by odor identification, odor discrimination, odor thresholds and retronasal perception of odors. The chemosensory event-related potentials, odor-induced changes of the electroencephalogram (EEG), the electroolfactogram and imaging techniques complete the olfactory function evaluation. Because olfaction has an important influence over the patients' quality of life, questionnaires are also used ${ }^{1,2}$.

In the USA, olfaction is evaluated mainly through psychophysical methods. Richard Doty ${ }^{3}$ states in an article that electrophysiological tests are often unreliable and influenced by chemical activation of non-olfactory intranasal fibers (e.g. trigeminal nerve free endings).

In Europe, there is a precise clinical workup procedure in order to assess both orthonasal and retronasal pathways. The perceptual and central nervous processing is different for each of them. Psychophysical evaluation methods, such as Sniffin'Sticks test or Smell Identification Test (UPSIT), are used for the orthonasal olfaction evaluation ${ }^{4,5}$.

Retronasal olfaction is assessed using a standardized method with a row of 20 items. The presented substances are common odors (condiments, food items available in powder). Squeezable plastic devices spread the powder in the oral cavity, in the middle of the tongue. The patient rinses his mouth after every sub- 
stance and the test procedure is 4 verbal items forced - choice $^{6}$.

According to the literature, advantages and drawbacks regarding psychophysical tests are certified. They are easy to implement, they are validated in multicenter studies and they have high test- retest reliability. But, at the same time, psychophysical tests are semi-objective, the results being influenced by the patient's bias, and they are not reliable in forensic patients $^{6,7}$.

Electrophysiological evaluation is used to register objective nervous responses after chemosensory olfactory stimulation.

It is hard to deliver odorants to the olfactory mucosa without registration of artefacts ${ }^{8}$, because the intranasal trigeminal system mediates the sensation of temperature, pressure, perception of nasal airflow during breathing, nociception, and also participates to the chemosensory perception of odorant stimuli.

An air - dilution olfactometer delivers pulses of odorants (2-phenylethanol) embedded within a constant airflow at a constant temperature and humidity and, at the same time, an EEG records the brain responses to the odorant stimuli.

The resulting EEG records one negative wave having a peak of 320 - $500 \mathrm{~ms}$ after the stimulus onset (N1) and a late positive wave (P2) with a peak of 450$800 \mathrm{~ms}$ after the stimulus onset ${ }^{9,10}$. According to Olofsson et al. and Hummel et al., a maximum peak of N1 recorded in the centro-parietal area represents an olfactory response, while in case of trigeminal stimuli, the maximum can be observed in the central area ${ }^{9,11}$.

Because olfactory event-related potentials (ERPs) usually exhibit a low signal-to-noise ratio, the clinical usefulness of ERPs has important limits, especially in the context of clinical diagnosis ${ }^{12}$.

Imaging examination also plays an important role in the olfactory function assessment. The most common examinations used in smell evaluation are the computer tomography scan (CT scan) and magnetic resonance imaging (MRI). The functional MRI (fMRI) efficiency in the field of olfaction is currently being studied.

The CT scan is helpful when olfactory cleft issues caused by rhinologic diseases are presumed to induce a smell condition. MRI is considered to be the first choice in the diagnosis of the olfactory impairment as it offers information about the olfactory bulb, the olfactory tract, the olfactory sulcus and the central olfactory areas.

Huart et al., in 2013, stated that the olfactory bulb has a particular importance in the study of the smell function, due to its variable volume depending on the background suffering. The capacity of the olfactory bulb to change its volume is called plasticity ${ }^{13}$.

Hummel et al. demonstrated that the volume of the olfactory bulb has an important significance in the determination of the olfactory disorders, as a decreased volume is encountered in hyposmia or anosmia caused by upper respiratory airways infections, head trauma, neurodegenerative disorders, congenital anosmia and psychiatric disease ${ }^{14-16}$.

In 2008, Buschhüter and his collaborators ${ }^{14}$ established normative values for the normal olfactory bulb volume. A value more than $58 \mathrm{~mm}^{3}$ should be encountered in people under the age of 45 , while people over 45 years should have an olfactory bulb volume over $46 \mathrm{~mm}^{3}$.

Functional MRI is being studied to be introduced as an evaluation mean of the hemodynamic response in brain areas, related to the activity of a neuronal population ${ }^{17}$. Brain structures examined during olfactory stimulation are the primary olfactory cortex (piriform cortex), the orbitofrontal cortex, the amygdala, the insular cortex, the cerebellum, thalamus and hypothalamus. At the moment, it is used only in research, because it seems that brain activation can appear even in anosmic patients ${ }^{17,18}$.

\section{OUR EXPERIENCE IN OLFACTOMETRIC ASSESSMENT}

Because in Romania a diagnosis protocol for the olfactory disturbances has not been established and because the necessary tools for an objective evaluation are not available, the olfactory function assessment together with the diagnosis and treatment of the smell disorders are still incomplete. In the ENT Clinic of "Sfanta Maria" Hospital, in Bucharest, starting from September 2015, a Center of Excellence for Research of Sensorial and Sensitive Disorders, Study of Infectoinflammatory, Tumoral and Obstructive Aero-digestive Pathology (CESITO) was founded due to a European funding programme contest. In our clinic, we assess the olfactory function using both subjective and objective methods. The smell threshold is determined using the TO 8 Olfactometer (dynamic olfactometry with nbutanol produced by Olfasense) and the Sensonic Snap and Sniff Test (Phenylethanol $\log 10$ dilution steps). The olfactometric assessment is completed by Natus Nicolet device, which registers the electrical activity of the brain during electrical stimulation of the olfactory mucosa. Having all these tools in our hands, our purpose is to develop a reliable evaluation and diagnosis protocol for the olfactory disturbances.

At present, we are undergoing a prospective study in our clinic, based on interdisciplinary approach because olfaction is a domain of interest not only for the ENT specialist, but also for other specialties. The purpose is to identify the cause and the background of the olfactory dysfunctions, and a strong collaboration with 
a radiologist, neurologist, neurosurgeon, forensic doctor or a psychiatrist is needed.

The inclusion criteria of the study are as following: patients with olfactory disturbances and suffering for obstructive rhinosinusal pathology, viral/ bacterial infectious pathology of the upper respiratory airways or head trauma, having history of professional exposure to pollutants and, of course, patients who agree to participate. Children, pregnant women and patients with psychiatric illnesses will be excluded.

The aims of our study are to establish smell disorders prevalence in general population and in ENT patients, to detect the olfactory function disorders impact upon patients' quality of life, to implement an evaluation protocol based on subjective and objective assessment methods, an adequate treatment identification and to ascertain an objective diagnosis protocol for patients with olfactory loss after head trauma (medico-legal cases).

In all patients, the olfactory function evaluation is based on a detailed history of the symptoms and patients' pathological background (i.e. infectious upper respiratory airways diseases, occupational exposure to pollutants, head trauma), on clinical and paraclinical examination (complete ENT examination, nasal endoscopy, culture of nasal secretions and imaging examination) and specific examination methods (subjective and objective).

The psychophysical evaluation is realised through the snap and sniff test and dynamic olfactometry (which uses different dilutions of n-butanol) to determine the smell threshold. These are subjective methods and they must be completed with electrophysiological tests represented by electric olfactory evoked potentials (EOEP).

The Natus Nicolet device registers the EOEP after electrical stimulation of the olfactory mucosa under endoscopic control. Five cutaneous electrodes are placed in the frontal and temporal regions, one reference electrode in the fronto-parietal area and one ground electrode (Figure 1). The maximum intensity of the current applied on the olfactory mucosa via the stimulation electrode (Figure 2) is $2 \mathrm{~mA}, 0.5 \mathrm{~ms} / \mathrm{stim}-$ ulation and we give approximate 300 stimulations according to the only one article referred to this subject ${ }^{19}$.

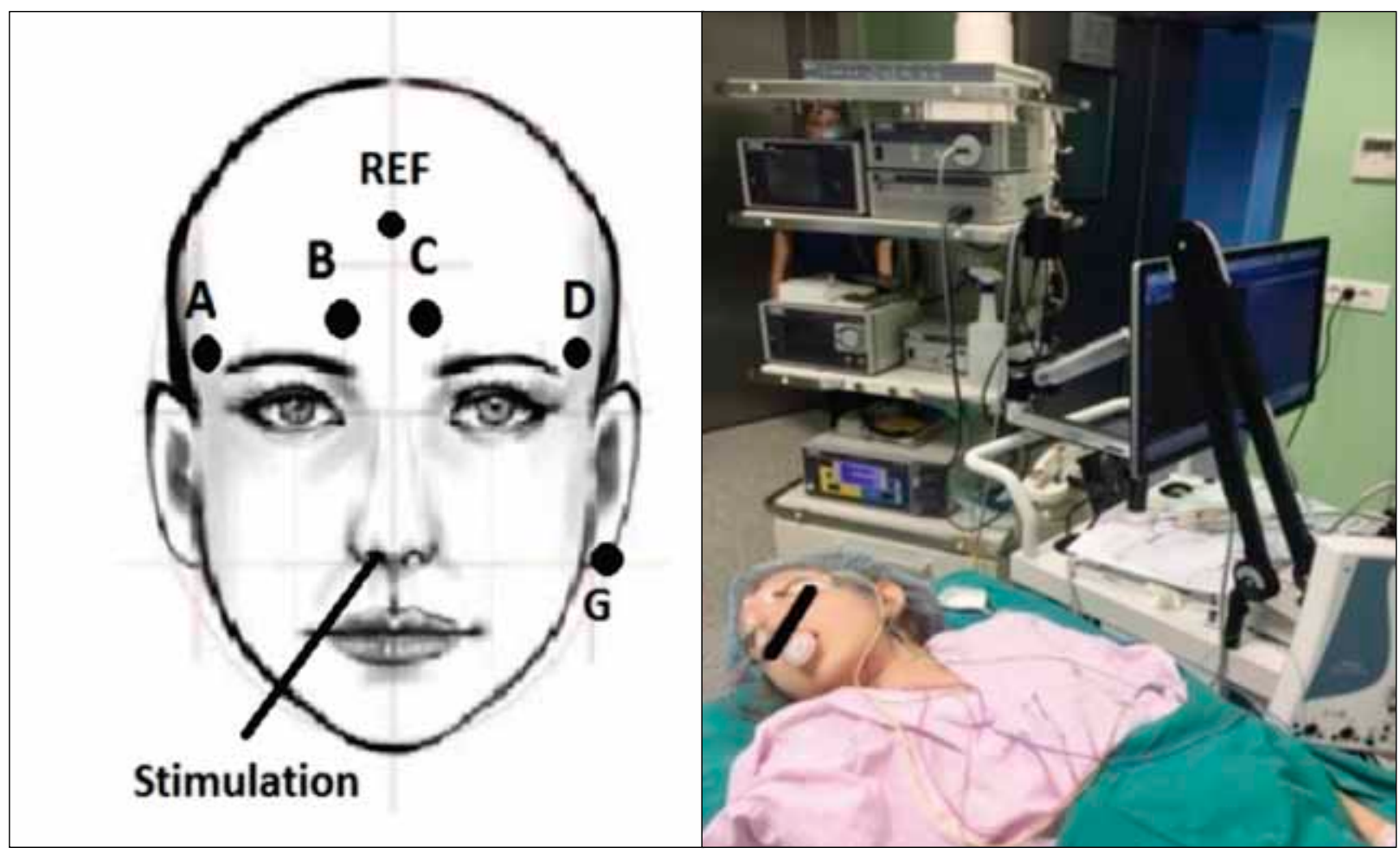

Figure 1 Electrodes placement for EOEP

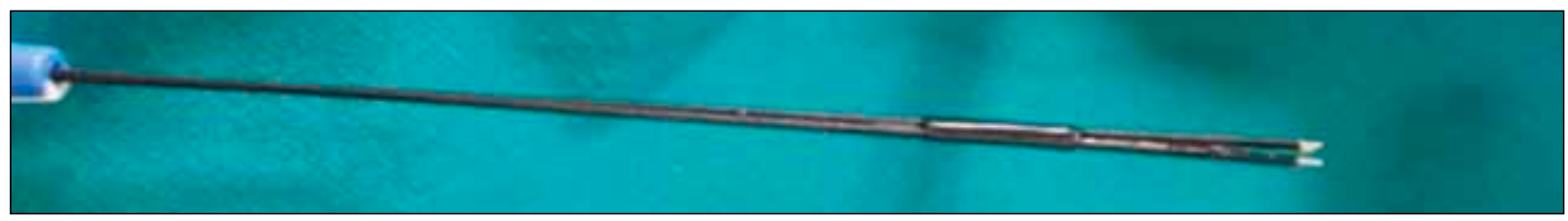

Figure 2 Electric stimulation electrode 
Unfortunately, we confront with some difficulties and limits in achieving our goals. As we all know, olfaction is a niche in the ENT field and scarce information regarding electric olfactory evoked potentials can be found. This challenged us to perform our study starting from a single article found in the literature, an article written by Ishimaru and his colleagues in $1997^{19}$.

The collaboration with a radiologist is also a difficulty, because the most important imaging examination goal is to measure the volume of the olfactory bulb $^{13}$. This can be realised only by a specialist with a good experience in this field.

Because in our country the insurance does not cover $100 \%$ of the costs of a CT or MRI, an economic burden for the patients may interfere.

Further, we present three of the most interesting cases of anosmia evaluated in our clinic.

Case 1. A 46-year-old female was admitted in our ENT Department in October 2015 for posttraumatic anosmia. According to the history of the patient, the symptomatology onset was in 2011 after a car accident. So, it was a medico- legal case.

The nasal endoscopic examination revealed right septal deviation in the upper portion, narrowing the olfactory area, but without obstructing it. There was no pathological aspect of the nasal or olfactory mucosa (Figure 3a; 3b).

The CT scan described a fracture line in the squamous part of the temporal bone. Olfactometric evaluation using dynamic olfactometry (one measurement consisting of 4 sequences) was performed and no smell perception was recorded irrespective of the nbutanol dilution presented (Figure 4).

We completed the investigation with the electric olfactory evoked potentials and we observed reduced amplitude of N1, and an increased latency with significantly decreased amplitude of $\mathrm{P} 2$. So, the results revealed no electric nervous activity in both primary and secondary olfactory cortices (Figure 5 ).

Corroborating the patient's history with the clinical and paraclinical investigations, the diagnosis of posttraumatic anosmia was confirmed.

Case 2. A 50-year-old male presented to the ENT Department of "Sfanta Maria" Hospital accusing subjective posttraumatic anosmia, installed after a physical aggression. This was also a case with legal implications. No pathological changes were found during the clinical ENT evaluation, but the CT scan showed a traumatic lesion of the cribriform plate (Figure 6).

The dynamic olfactometry was also assessed for this patient (one measurement consisting of 4 sequences), with no response registered (Figure 7), but he did not consent to perform the electric olfactory evoked potentials.

In conclusion, we could not objectivise patient's complaints because of his lack of compliance. In this situation, malingering might be suspected, but it cannot be demonstrated while the patient did not accept to complete the olfactory evaluation.
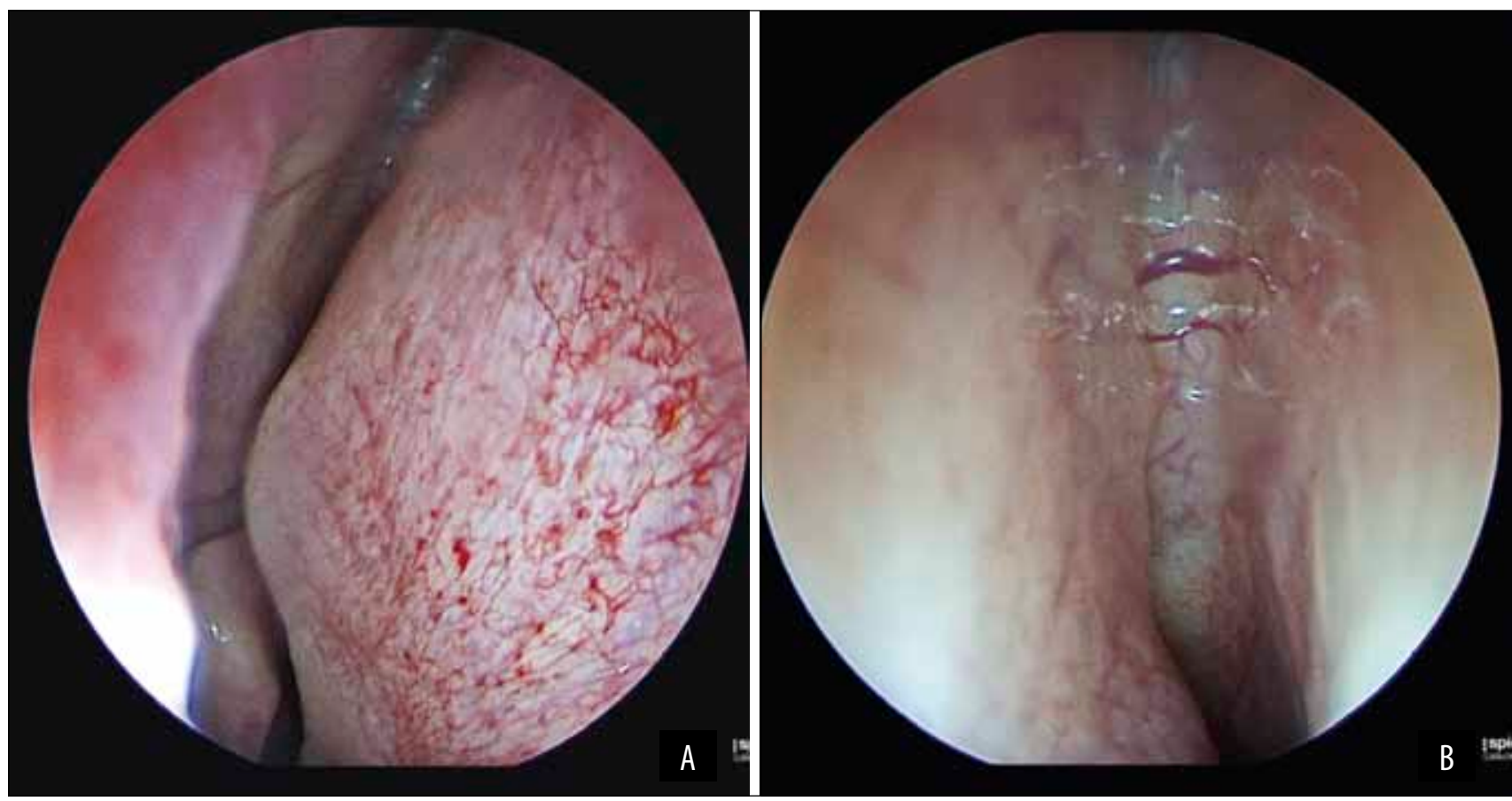

Figure 3 Intranasal endoscopic aspect - right septal deviation in the upper portion, narrowing the olfactory area, but without obstructing it (A); normal aspect of the olfactory mucosa (B) 


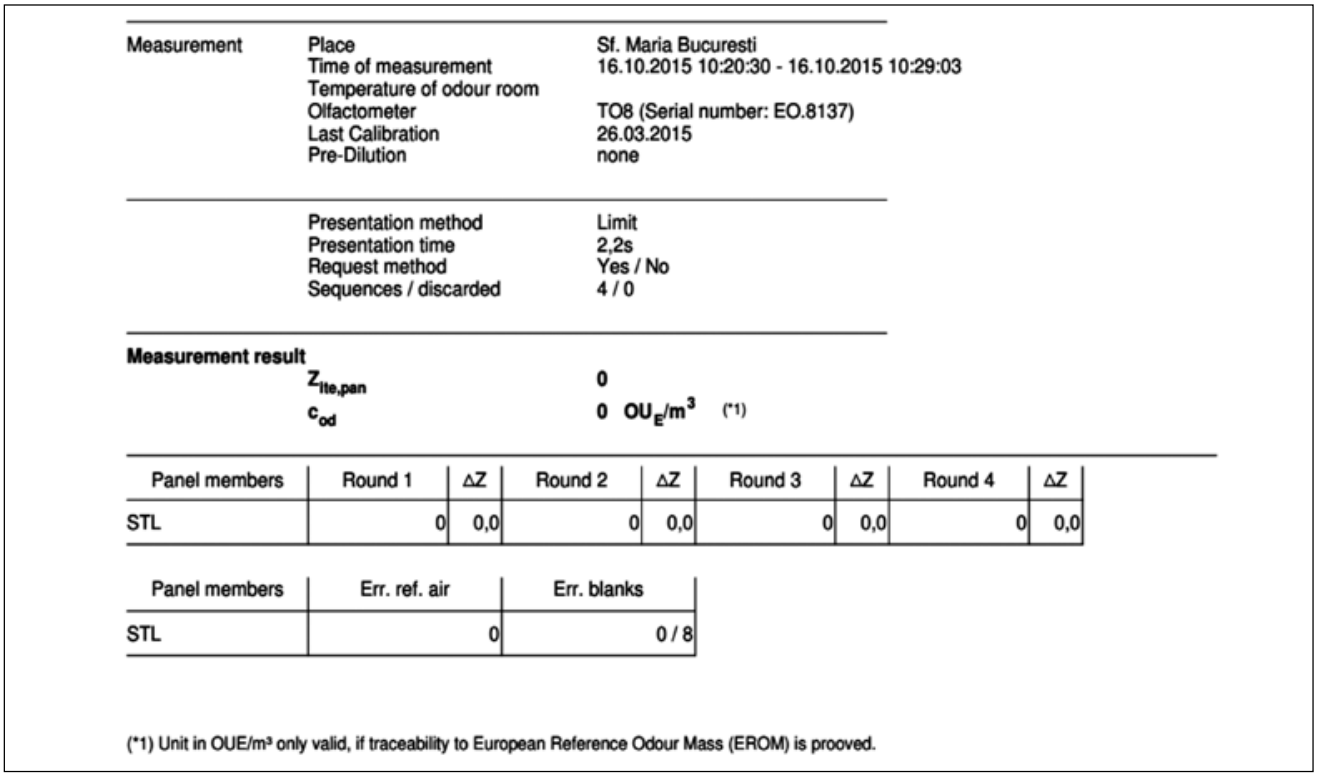

Figure 4 Dynamic olfactometry (n-Butanol): no smell perception

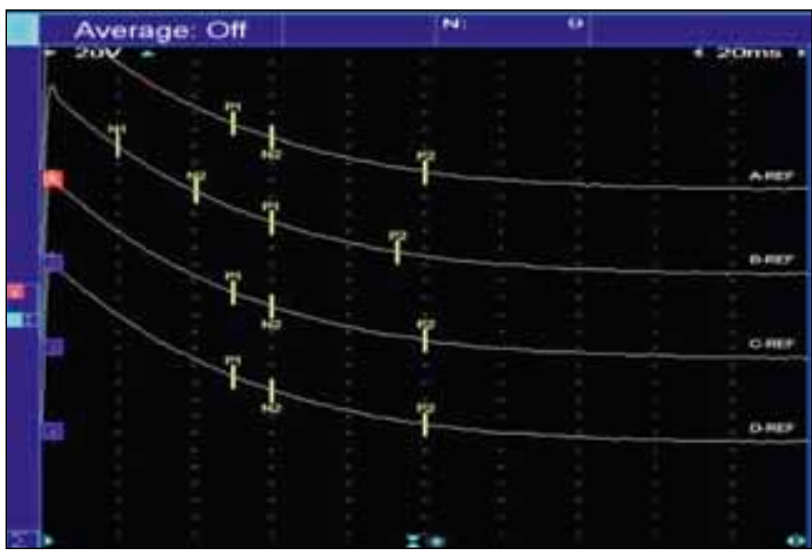

Figure 5 Electric Olfactory Evoked Potentials result - reduced amplitude of $\mathrm{N} 1$, and an increased latency with significantly decreased amplitude of P2

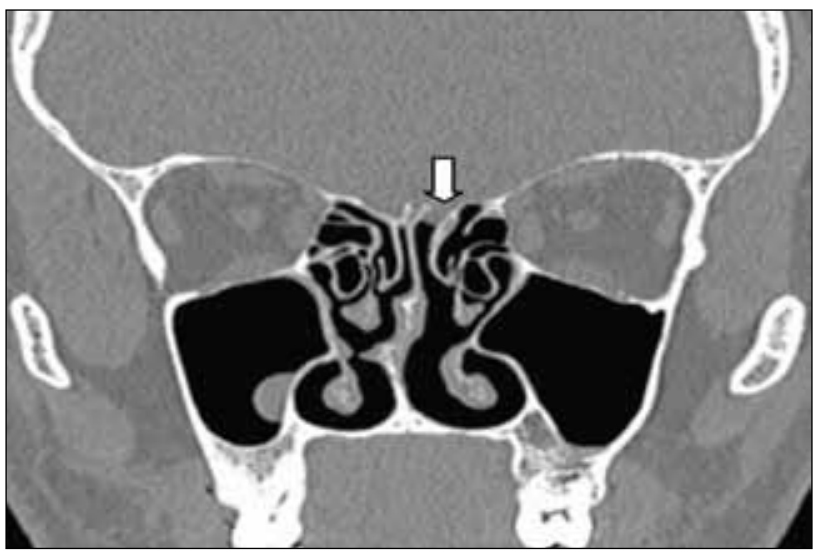

Figure 6 Cranial CT scan (coronal slice). Arrow showing the cribriform plate defect

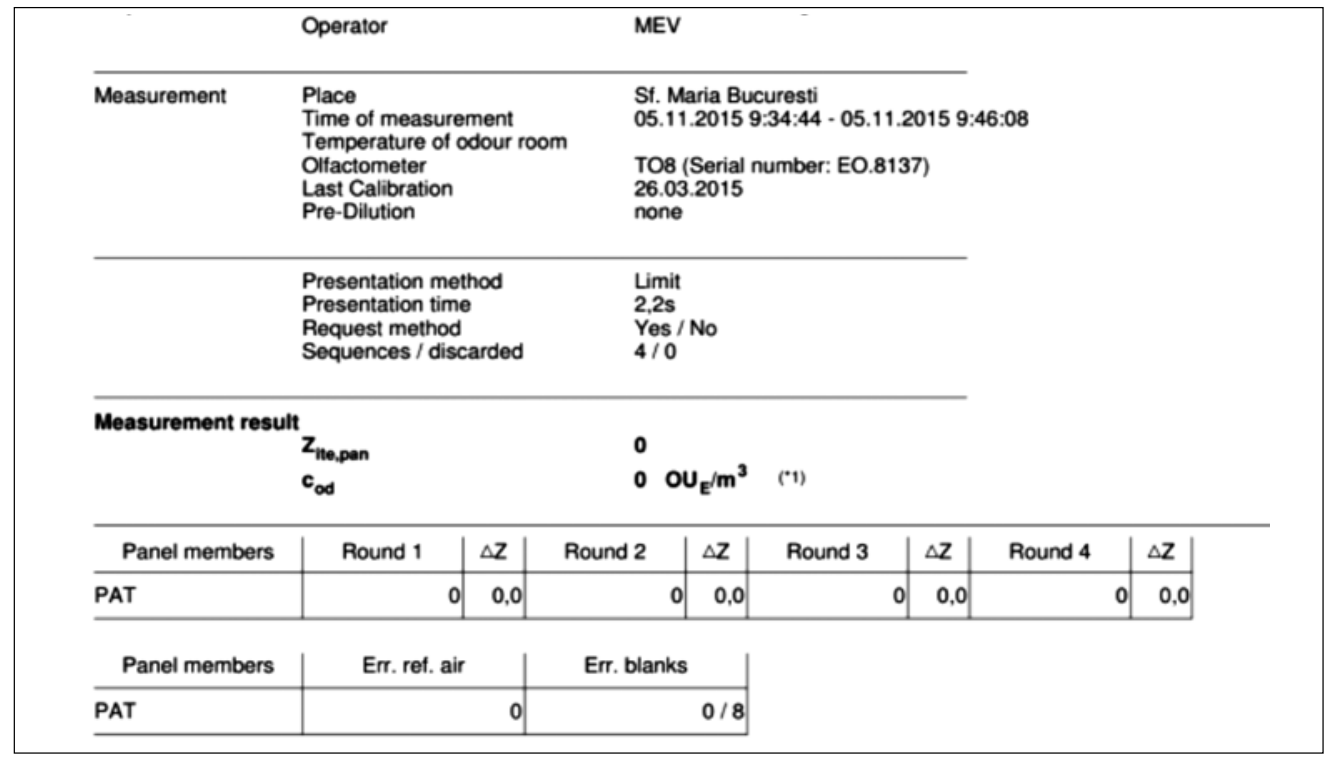

Figure 7 Dynamic olfactometry result - no response registered 
Case 3. A 7-year-old little girl was referred by her parents to our clinic because she had been complaining about the lack of sense of smell. Neither she nor her parents could indicate the onset of her subjective anosmia. She had no significant personal history (trauma, severe upper respiratory airways infections). A complete clinical and paraclinical evaluation was performed: the ENT examination revealed an unob-

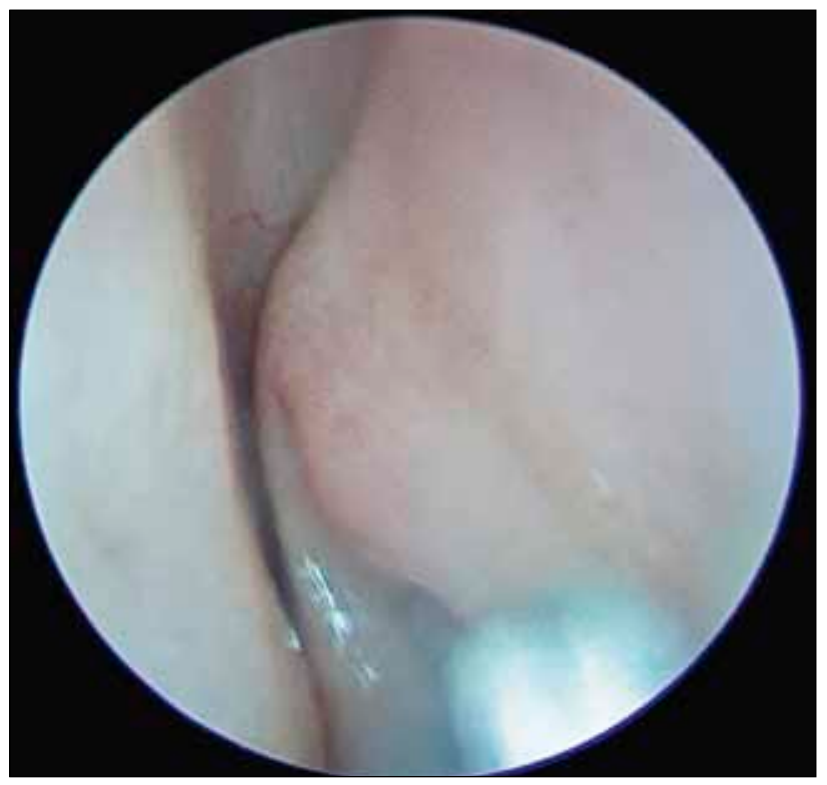

Figure 8 Endonasal aspect (endoscopic view) - unobstructive right nasal septum deviation and hypertrophic inferior nasal turbinates structive right nasal septum deviation and hypertrophic inferior nasal turbinates (Figure 8), while the CT scan was normal (Figure 9).

A dynamic olfactometry, one measurement consisting of 2 sequences, was registered; there was no response from the patient (Figure 10).

The parents also agreed to perform the electric olfactory evoked potentials under general anaesthesia.

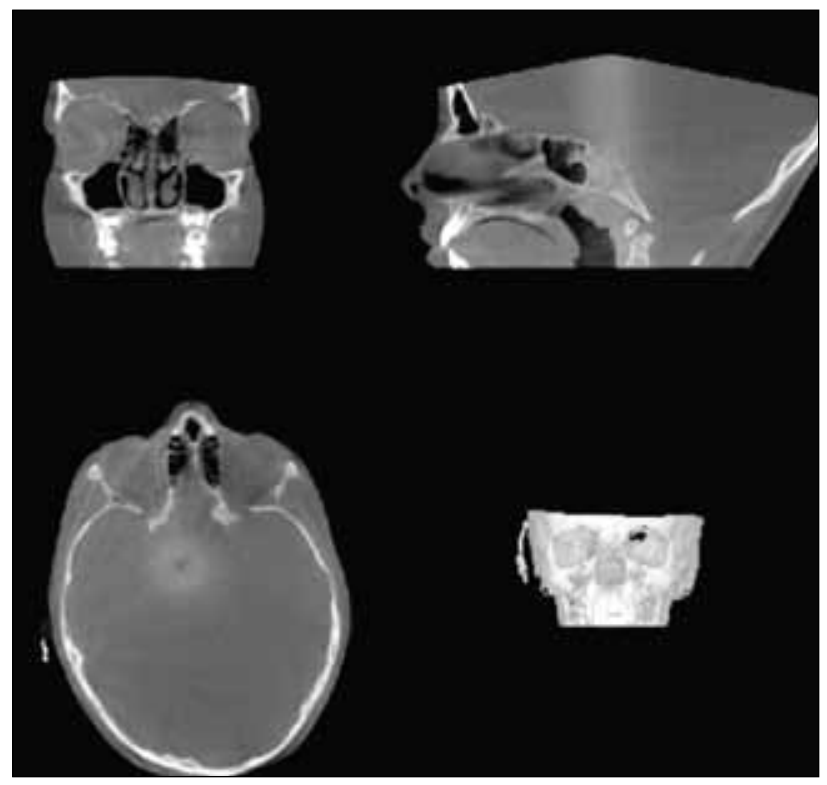

Figure 9 Cranio - facial CT scan with normal aspect

\begin{tabular}{|c|c|c|}
\hline Samplo & \multicolumn{2}{|r|}{ n-Butanol } \\
\hline Propect & $\begin{array}{l}\text { Name } \\
\text { Operator }\end{array}$ & \\
\hline \multirow[t]{2}{*}{ Measurement } & $\begin{array}{l}\text { Place } \\
\text { Time of measurement } \\
\text { Temperature of ocour room } \\
\text { Ollactometer } \\
\text { Last Calibration } \\
\text { Pro-Dilution }\end{array}$ & $\begin{array}{l}\text { St Marla Bucurnesti } \\
\text { O8.06.2016 14:20.08 - 08.06.2016 14:26:02 } \\
\text { TO8 (Seral number: EO.8137) } \\
\text { 26.03.2015 } \\
\text { ncne }\end{array}$ \\
\hline & $\begin{array}{l}\text { Presentation method } \\
\text { Presentation bine } \\
\text { Request method } \\
\text { Sequences / dscarded }\end{array}$ & $\begin{array}{l}\text { Limit } \\
2.2 \mathrm{~s} \\
\text { Yes / No } \\
2: 0\end{array}$ \\
\hline Measurement resul & $z_{\text {nopen }}$ & $\begin{array}{lll}0 & \\
0 & O U_{E} / m^{3} & (1)\end{array}$ \\
\hline Panel members & Round 1 $|\Delta Z|$ & Found $2|\Delta z|$ \\
\hline $\mathrm{CID}$ & 00,0 & \begin{tabular}{l|l|}
0 & 0,0 \\
\end{tabular} \\
\hline Panel members & Err. ret. air & Err. blanks \\
\hline CID & 0 & $0 / 4$ \\
\hline
\end{tabular}

Figure 10 Dynamic olfactometry result with no response 


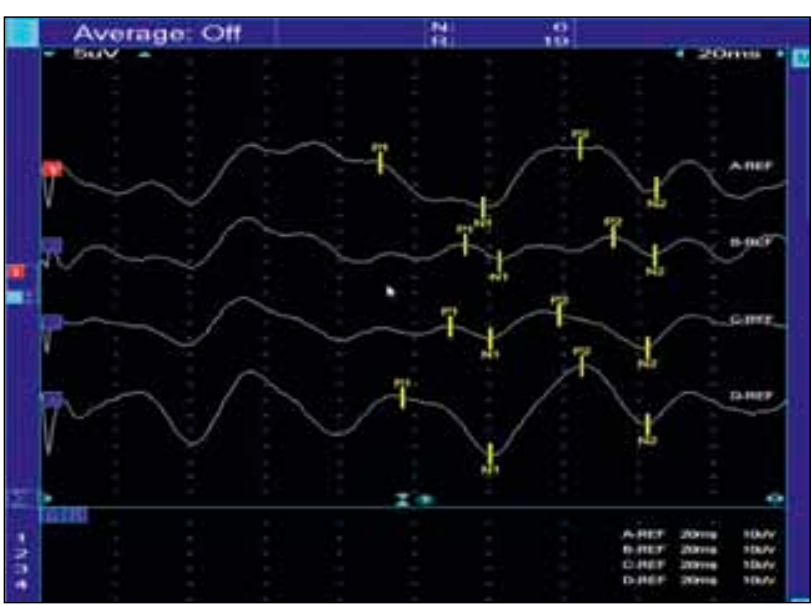

Figure 11 Olfactory evoked potentials - normal activity of the olfactory nerve

The investigation was performed using endoscopic control in order to give an accurate stimulation of the olfactory mucosa and normal activity of the olfactory nerve was observed (Figure 11).

The particularity of this case consists in the discrepancy between the results obtained in the dynamic olfactometry and electric olfactory evoked potentials. This contradiction makes us believe that she is a malingerer, giving also the integrity of her sense of taste, as she stated that her favourite desert is forest fruits ice cream. Summing the personal history of the patient and the paraclinical evaluations, we could not confirm the diagnosis of anosmia.

Considering the difficulty of working with children, because of their scarce compliance, this was a tough case and the patient must be revaluated when she gets older. Children's lack of collaboration is all the more reason why we do not include them in the undergoing study.

\section{MALINGERING OR HOW TO MANAGE MEDICO-LEGAL CASES}

Because the olfactory dysfunction following head trauma is currently compensable, it is mandatory to identify malingerers. Using only subjective evaluation methods, there is a strong possibility that the patient is out to deceive the analyst by pretending malingering in the case of the existence or nonexistence of post-traumatic impaired smell ${ }^{20}$.

In Europe, the diagnosis protocol includes all means of evaluation: subjective - tests for threshold determination, identification and discrimination and objective - event- related olfactory evoked potentials ${ }^{21}$. The final diagnosis is established after correlating the patient's history, psychophysical tests and the electrophysiological evaluation.

According to Richard Doty ${ }^{3}$, there is no need for electrophysiological assessment, because when measure- ment is realised with the Smell Identification Test (UPSIT), improbable responding on forced-choice chemosensory tests detects malingering. Even in case of anosmia, $25 \%$ of the stimuli in a four-alternative test should be correctly identified and the probability of obtaining a score of 0 on the UPSIT and not avoiding the correct responses is around 1 in $100,000^{22}$.

\section{CONCLUSIONS}

In conclusion, we are still far away from a complete knowledge in the olfaction field. Different points of view regarding an accurate assessment can be found in the literature and no worldwide accepted evaluation protocol is available.

In Romania, the olfactory function evaluation is a niche in the ENT specialty and we try to develop it as much as we can. We need to follow the European model for the assessment protocol, because the forensic doctors require also objective evidence of the smell impairment in order to give a correct solution to legal cases.

Conflict of interest: The authors have no conflict of interest.

Contribution of authors: All authors have equally contributed to this work.

\section{REFERENCES}

1. Scadding G., Hellings P., Alobid I., et al. - Diagnostic tools in Rhinology EAACI position paper. Clin Transl Allergy., 2011;29(2):289-292. doi: 10.1186/2045-7022-1-2.

2. Croy I., Buschhüter D., Seo H.S., Negoias S., Hummel T. - Individual significance of olfaction: development of a questionnaire. Eur Arch Otorhinolaryngol., 2010;267(1):67-71. doi: 10.1007/s00405-009-1054-0.

3. Doty R.L. - Olfactory dysfunction and its measurement in the clinic and workplace. Int Arch Occup Environ Health, 2006;79(4):268-282. Epub 2006 Jan 21.

4. Hummel T., Heilmann S., Landis B.N., Reden J., Frasnelli J., Small D.M., Gerber J. - Perceptual differences between chemical stimuli presented through the ortho- or retronasal route. Flavour Fragr. J., 2006;21(1):4247 .

5. Small D.M., Gerber J.C., Mak Y.E., Hummel T. - Differential neural responses evoked by orthonasal versus retronasal odorant perception in humans. Neuron., 2005;47(4):593-605.

6. Heilmann S., Strehle G., Rosenheim K., Damm M., Hummel T. - Clinical assessment of retronasal olfactory function. Arch Otolaryngol Head Neck Surg., 2002;128(4):414-418.

7. Doty R.L., Shaman P., Kimmelman C.P., Dann M.S. - University of Pennsylvania Smell Identification Test: a rapid quantitative olfactory function test for the clinic. Laryngoscope, 1984;94(2 Pt 1):176-178.

8. Moncrief R.W. - Effect of odours on EEG records. Perfumery essential oil Rec, 1962;53:727-760.

9. Hummel T., Genow A., Landis B.N. - Clinical assessment of human gustatory function using event related potentials. J Neurol Neurosurg 
Psychiatry., 2010;81(4):459-464. doi: 10.1136/jnnp.2009.183699. Epub 2009 Sep 1.

10. Haehner A., Gruenewald G., Dibenedetto M., Hummel T. - Responses to olfactory and intranasal trigeminal stimuli: relation to the respiratory cycle. Neuroscience, 2011;175:178-183. doi: 10.1016/j.neuroscience.2010.11.038. Epub 2010 Dec 9.

11. Olofsson J.K., Broman D.A., Gilbert P.E., Dean P., Nordin S., Murphy C. - Laterality of the olfactory event-related potential response. Chem Senses., 2006;31 (7):699-704. Epub 2006 Jul 18.

12. Rombaux P., Mouraux A., Bertrand B., Guerit J.M., Hummel T. Assessment of olfactory and trigeminal function using chemosensory event-related potentials. Neurophysiol Clin., 2006;36(2):53-62. Epub 2006 Apr 19.

13. Huart C., Rombaux P., Hummel T. - Plasticity of the human olfactory system: the olfactory bulb. Molecules, 201;18(9);11586-11600. doi: 10.3390/molecules180911586.

14. Buschhüter D., Smitka M., Puschmann S., Gerber J.C., Witt M., Abolmaali N.D., Hummel T. - Correlation between olfactory bulb volume and olfactory function. Neuroimage, 2008;42(2):498-502. doi: 10.1016/j.neuroimage.2008.05.004. Epub 2008 May 10.

15. Hummel T., Smitka M., Puschmann S., Gerber J.C., Schaal B., Buschhüter
D. - Correlation between olfactory bulb volume and olfactory function in children and adolescents. Exp Brain Res., 2011;214(2):285-291. doi: 10.1007/s00221-011-2832-7. Epub 2011 aug 13.

16. Turetsky B.I., Moberg P.J., Yousem D.M., Doty R.L., Arnold S.E., Gur R.E. - Reduced olfactory bulb volume in patients with schizophrenia. Am J Psychiatry., 2000;157(5):828-830.

17. Sobel N., Prabhakaran V., Hartley C.A., Desmond J.E., Glover G.H., Sullivan E.V., Gabrieli J.D. - Blind smell: brain activation induced by an undetected air-borne chemical. Brain, 1999;122(Pt 2):209-217.

18. Zatorre R.J., Jones-Gotman M., Evans A.C., Meyer E. - Functional localization and lateralization of human olfactory cortex. Nature, 1992;360(6402):339-340.

19. Ishimaru T., Shimada T., Sakumoto M., Miwa T., Kimura Y., Furukawa M. - Olfactory evoked potential produced by electrical stimulation of the human olfactory mucosa. Chem Senses., 1997;22(1):77-81.

20. Mann N.M. - Head injury and anosmia. Conn Med., 2003;67(9):545-547.

21. Collet S., Grulois V., Bertrand B., Rombaux P. - Post-traumatic olfactory dysfunction: a cohort study and update. B-ENT, 2009;5 Suppl 13:97-107.

22. Doty R.L., Crastnopol B. - Correlates of chemosensory malingering. Laryngoscope, 2010;120(4):707-711. doi: 10.1002/lary.20827. 